\title{
The Gravity Gauge Theory and Gravity Field Equation in Flat Space
}

\author{
Xiangyao Wu, Benshan Wu, Jiping Liu, Xiaojing Liu, Yumeng Guo, Qing Pan, Ji Ma, Xiaoru Zhang, \\ Han Liu
}

Institute of Physics, Jilin Normal University, Siping, China

Email: wuxy65@126.com

How to cite this paper: $\mathrm{Wu}, \mathrm{X} . \mathrm{Y}$., $\mathrm{Wu}$, B.S., Liu, J.P., Liu, X.J., Guo, Y.M., Pan, Q., Ma, J., Zhang, X.R. and Liu, H. (2018) The Gravity Gauge Theory and Gravity Field Equation in Flat Space. Journal of High Energy Physics, Gravitation and Cosmology, 4, 567-580.

https://doi.org/10.4236/jhepgc.2018.43034

Received: April 11, 2018

Accepted: July 28, 2018

Published: July 31, 2018

Copyright $\odot 2018$ by authors and Scientific Research Publishing Inc. This work is licensed under the Creative Commons Attribution International License (CC BY 4.0).

http://creativecommons.org/licenses/by/4.0/

\begin{abstract}
In this paper, we have proposed the theory of $U(1)$ gravity gauge, and the gravity theory has been introduced into quantum field theory. We have further given the tensor equation of gravity field in the flat space, and found the gravity field equation is the Lorentz covariant and gauge invariant. The gravity theory can be quantized and can be unified with the electroweak and strong interaction at a new gauge group $U(1) \otimes S U(2) \otimes S U(3)$.
\end{abstract}

\section{Keywords \\ Gravity Field, General Relativity, $U(1)$ Gauge Theory, Gravity Field Equation}

\section{Introduction}

The Einstein's general theory of gravity (GR) is treated as geometry of curved space-time, which appears to provide a successful macroscopic description of all known gravitational phenomena; it is of interest to explore alternative theories that may provide a more fundamentally appealing description or suggest new experiments leading to the discovery of new phenomena [1] [2]. As well known, most of the fundamental interactions in nature based on the gauge theory have been constructed to be physically sensible due to that they lead to the consistent quantum description. The gauge theories are in principle applicable up to arbitrarily high energy scales. Thus, it would be natural to seek a gauge theory structure for the gravitational interaction in which the general relativity is derived as the low energy limit. Quantum gauge theory of gravity is proposed in the framework of quantum field theory, the mathematical structures of GR in the first-order formalism and gauge theory appear very similar at classical level 
and for this reason, in the MacDowell-Mansouri theory, general theory of gravity was reformulated as a Yang-Mills theory [3]-[8].

Developing of a consistent quantum theory of the gravity at very small distances or high energy scales, has been interested by many physicists in which the gravity is strongly played by the rules of the quantum mechanics. In fact, a theory of the gravity is to study the space-time. Since well understanding of the quantum gravity allows us to deal with the behavior of the space-time at very small distances. The main motivations for seeking this are just purely theoretical because of the fact that there have not had immediately experimental observations for the quantum gravitational effects so far. However, some indirect experimental constraints for Planck-length quantum properties of the space-time have been exploited with a growing number of studies [9]-[16]. In Ref. [17], the authors have taken into consideration a generalization of gauge theories based on the analysis of the structural characteristics of Maxwell theory, which is based on few principles related to different orders of commutators between covariant derivatives. They have modified theory of gravity, in which the algebra of operators of covariant derivatives leads to an additional term in the equation of motion associated with the non-conservation of the energy-momentum tensor.

In this paper, we have proposed the gauge theory of gravity. In Dirac equation and $K-G$ equation, they have introduced the vector gauge field, such as electroweak and strong interaction gauge field, which are vector gauge fields, and have not introduced the gravity gauge field. In order to introduce the gravity gauge field, we should add a term of partial derivative $\partial_{\mu} \partial_{\nu}$ in Dirac equation and $K-G$ equation. At the $U(1)$ gauge transformation, the gravity gauge field should be introduced naturally. Otherwise, we give the equation of gravity tensor field at the flat Minkowski spacetime, and further prove the gravity field equation is the Lorentz covariant and gauge invariant. The gravity theory can be quantized and can be unified with the electroweak and strong interaction at a new gauge group $U(1) \otimes S U(2) \otimes S U(3)$.

\section{The $U(1)$ Gauge Field of Gravity}

The Einstein gravitational field equation with matter is

$$
R_{\mu v}-\frac{1}{2} g_{\mu v} R=\frac{8 \pi G}{c^{4}} T_{\mu v},
$$

where $T_{\mu v}$ is the energy-momentum tensor, $R_{\mu v}$ is the Ricci tensor, $R$ is the Ricci scalar curvature, $g_{\mu v}(x)$ is the symmetric metric tensor, $G$ is Newton's gravitational constant and $c$ is the speed of light. The energy-momentum tensor $T_{\mu v}$ gives the motion and distribution of the matter in spacetime, producing the gravitation field, and $R_{\mu \nu}-\frac{1}{2} g_{\mu v} R$ is the geometry tensor of Riemannian curved spacetime. The important properties of the Einstein gravitational field equation are: the distribution and motion of the matter determine the curved spacetime structure, and the distribution of matter can be determined by the 
spacetime structure.

The gravitational field is the geometry field of spacetime, which is equivalent to the spacetime curve. The general theory of gravity can be reinterpreted as a field theory over flat Minkowski spacetime, the gravity can be taken as real matter field, produced by the matter motion and distribution. In the following, we shall introduce gravity field in the Minkowski spacetime, which is massless spin-2 field. In group theory, the representation of the rotation group and Lorentz group is described by a field with $2 s+1$ independent components for a particle with spin $s=0,1,2, \cdots$. For the massless spin-2 gravity field, it should be described by a tensor field $\phi^{\mu v}(x)$ of second rank.

In quantum field theory, the Lagrangean density of real field, complex field and Dirac spinor field are:

$$
\begin{aligned}
& L=\frac{1}{2}\left(\partial_{\mu} \phi \partial^{\mu} \phi-m^{2} \phi^{2}\right), \\
& L=\partial_{\mu} \phi^{+} \partial^{\mu} \phi-m^{2} \phi^{+} \phi, \\
& L=\bar{\psi}\left(i \gamma^{\mu} \partial_{\mu}-m\right) \psi .
\end{aligned}
$$

At Equations (2), (3) and (4), if the local gauge is invariant, the gauge field should be introduced. We need form a gauge covariant derivation $D_{\mu}$ to replace $\partial^{\mu}$, it is

$$
\partial_{\mu} \rightarrow D_{\mu}=\partial_{\mu}+A_{\mu}(x),
$$

where $A_{\mu}(x)=-i g A_{\mu}^{a}(x) T^{a}$ is gauge field. For the electroweak interaction theory, it has the $S U(2) \otimes U(1)$ gauge symmetry, and their gauge fields are the linear combination of fields $\vec{A}_{\mu}(x)$ and $B_{\mu}(x)$, they are

$$
\begin{gathered}
W_{\mu}^{-}=\frac{1}{\sqrt{2}}\left(A_{\mu}^{1}+i A_{\mu}^{2}\right), \\
W_{\mu}^{+}=\frac{1}{\sqrt{2}}\left(A_{\mu}^{1}-i A_{\mu}^{2}\right), \\
Z_{\mu}=\frac{1}{\sqrt{g^{2}+g^{\prime 2}}}\left(g A_{\mu}^{3}-g^{\prime} B_{\mu}\right), \\
A_{\mu}=A_{\mu}^{3} \sin \theta_{\omega}+B_{\mu} \cos \theta_{\omega} .
\end{gathered}
$$

These gauge fields are the vector fields with spin $s=1$, and corresponding to the particles $W_{\mu}^{-}, W_{\mu}^{+}, Z_{\mu}$ and $\gamma$.

For the strong interaction theory of between quark and gluon, it has the $S U$ (3) gauge symmetry, and the gauge field are eight gluons vector fields $B_{\mu}^{a}(a=1,2, \cdots, 8)$. In quantum gauge theory, the all gauge fields are vector fields with spin $s=1$, which can only describe electromagnetic interaction, weak interaction and strong interaction, and cannot describe the gravity interaction with spin $s=2$. Why the quantum gauge theory cannot describe the gravity interaction? From Equation (2) to (4), we can find the differential operator $\partial_{\mu}$ appears alone in the Lagrangean density. When we make the $U(1)$ local gauge 
transformation:

$$
\begin{aligned}
& \psi(x) \rightarrow \psi^{\prime}(x)=\mathrm{e}^{-i \gamma(x)} \psi(x), \\
& \bar{\psi}(x) \rightarrow \bar{\psi}^{\prime}(x)=\mathrm{e}^{-i \gamma(x)} \bar{\psi}(x),
\end{aligned}
$$

the $\partial_{\mu}$ should be make the transformation as Equation (5), it only introduce the vector gauge field $A_{\mu}(x)$. If we add the term $\partial_{\mu} \partial_{v}$ to Equations (2), (3) and (4), the $\partial_{\mu}$ and $\partial_{\mu} \partial_{\nu}$ should be made the transformations

$$
\begin{aligned}
\partial_{\mu} & \rightarrow D_{\mu}=\partial_{\mu}+A_{\mu}(x), \\
\partial_{\mu} \partial_{v} & \rightarrow D_{\mu v}=\partial_{\mu} \partial_{v}+i g \phi_{\mu v}(x),
\end{aligned}
$$

where $A_{\mu}(x)$ is the vector gauge field with spin $s=1$, and $\phi_{\mu v}(x)$ is the tensor gauge field with spin $s=2$, i.e., the gravity gauge field.

For the Dirac equation

$$
\left(i \gamma^{\mu} \partial_{\mu}-m\right) \psi=0,
$$

we add the term $\partial_{\mu} \partial_{v}$, it becomes

$$
\left(i \gamma^{\mu} \partial_{\mu}-m-\frac{\varepsilon}{m} \gamma^{\mu} \gamma^{\mu} \partial_{\mu} \partial_{v}\right) \psi=0,
$$

where $\varepsilon$ is the dimensionless constant. Since the gravity is very weak, the contribution of new added term $\frac{\varepsilon}{m} \gamma^{\mu} \gamma^{\mu} \partial_{\mu} \partial_{v}$ should be very small, there is

$$
\varepsilon \ll m^{2},
$$

the Lagrangean density of Equation (15) is

$$
L=\bar{\psi}\left(i \gamma^{\mu} \partial_{\mu}-m-\frac{\varepsilon}{m} \gamma^{\mu} \gamma^{\mu} \partial_{\mu} \partial_{v}\right) \psi
$$

then

$$
\begin{gathered}
\frac{\partial L}{\partial \bar{\psi}}=-m \psi+i \gamma^{\mu} \partial_{\mu} \psi-\frac{\varepsilon}{m} \gamma^{\mu} \gamma^{\mu} \partial_{\mu} \partial_{\nu} \psi, \\
\partial_{\mu} \frac{\partial L}{\partial\left(\partial_{\mu} \bar{\psi}\right)}=0,
\end{gathered}
$$

substituting Equations (18) and (19) into the Lagrangean equation

$$
\frac{\partial L}{\partial \bar{\psi}}-\partial_{\mu} \frac{\partial L}{\partial\left(\partial_{\mu} \bar{\psi}\right)}=0
$$

we can obtain the Equation (15). At the U(1) local gauge transformations (10) and (11), in order to make Equation (17) gauge invariant, we should introduce the gauge fields $A_{\mu}$ and $\phi_{\mu v}$, they are

$$
\begin{gathered}
\partial_{\mu} \rightarrow D_{\mu}=\partial_{\mu}+i e A_{\mu}, \\
\partial_{\mu} \partial_{v} \rightarrow D_{\mu v}=\partial_{\mu} \partial_{v}+i g \phi_{\mu v},
\end{gathered}
$$

where $e$ and $g$ are the coupling constants, and the Equation (17) becomes 


$$
L=\bar{\psi}\left(i \gamma^{\mu} D_{\mu}-m-\frac{\varepsilon}{m} \gamma^{\mu} \gamma^{\mu} D_{\mu v}\right) \psi=L_{e}+L_{m},
$$

where

$$
\begin{aligned}
& L_{e}=\bar{\psi}\left(i \gamma^{\mu} D_{\mu}-m\right) \psi, \\
& L_{m}=-\frac{\varepsilon}{m} \bar{\psi} \gamma^{\mu} \gamma^{\mu} D_{\mu \nu} \psi,
\end{aligned}
$$

at $U(1)$ gauge transformations (10) and (11) the Lagrangean density $L$ should be changed into $L^{\prime}$, it is

$$
L^{\prime}=\bar{\psi}^{\prime}\left(i \gamma^{\mu} D_{\mu}^{\prime}-m-\varepsilon m \gamma^{\mu} \gamma^{v} D_{\mu \nu}^{\prime}\right) \psi^{\prime}=L_{e}^{\prime}+L_{m}^{\prime},
$$

where

$$
\begin{aligned}
& L_{e}^{\prime}=\bar{\psi}^{\prime}\left(i \gamma^{\mu} D_{\mu}^{\prime}-m\right) \psi^{\prime}, \\
& L_{m}^{\prime}=-\frac{\varepsilon}{m} \bar{\psi}^{\prime} \gamma^{\mu} \gamma^{v} D_{\mu \nu}^{\prime} \psi^{\prime},
\end{aligned}
$$

the covariant derivatives $D_{\mu}$ and $D_{\mu v}$ should be transformed as

$$
\begin{gathered}
D_{\mu} \rightarrow D_{\mu}^{\prime}=\partial_{\mu}+i e A_{\mu}^{\prime}, \\
D_{\mu v} \rightarrow D_{\mu v}^{\prime}=\partial_{\mu} \partial_{v}+i g \phi_{\mu v}^{\prime},
\end{gathered}
$$

the local gauge invariance demand the Lagrangean density is invariable, i.e., $L=L^{\prime}$.

At transformation

$$
A_{\mu} \rightarrow A_{\mu}^{\prime}=A_{\mu}+\frac{1}{e} \partial_{\mu} \gamma(x)
$$

these is

$$
L_{e}^{\prime}=L_{e}
$$

then we require

$$
L_{m}^{\prime}=L_{m}
$$

i.e.,

$$
\bar{\psi}^{\prime} \gamma^{\mu} \gamma^{v} D_{\mu \nu}^{\prime} \psi^{\prime}=\bar{\psi} \gamma^{\mu} \gamma^{v} D_{\mu \nu} \psi
$$

by Equation (34), we can give the gravity gauge transformation at $U(1)$ gauge group. As

$$
\begin{aligned}
& \partial_{\mu} \partial_{\nu}\left(\mathrm{e}^{-i \gamma(x)} \psi\right)=\partial_{\mu}\left(\mathrm{e}^{-i \gamma(x)} \psi\left(-i \partial_{\mu} \gamma(x) \psi+\mathrm{e}^{-i \gamma(x)} \partial_{\nu} \psi\right)\right) \\
& =\mathrm{e}^{-i \gamma(x)}\left(-\partial_{\mu} \gamma(x) \partial_{\nu} \gamma(x)-i \partial_{\nu} \gamma(x) \partial_{\mu}-i \partial_{\mu} \partial_{\nu} \gamma(x)-i \partial_{\mu} \gamma(x) \partial_{\nu}+\partial_{\mu \nu}\right) \psi,
\end{aligned}
$$

the Equation (34) becomes

$$
\begin{aligned}
& \bar{\psi}^{\prime} \gamma_{\mu} \gamma_{\nu} D_{\mu \nu}^{\prime} \psi^{\prime}=\bar{\psi} \mathrm{e}^{+i \gamma(x)} \gamma_{\mu} \gamma_{\nu}\left(\partial_{\mu} \partial_{v}+i g \varphi_{\mu \nu}^{\prime}\right) \mathrm{e}^{-i \gamma(x)} \psi \\
& =\bar{\psi} \gamma^{\mu} \gamma^{\nu}\left(-\partial_{\mu} \gamma(x) \partial_{\nu} \gamma(x)-i \partial_{\nu} \gamma(x) \partial_{\mu}-i \partial_{\mu} \partial_{\nu} \gamma(x)-i \partial_{\mu} \gamma(x) \partial_{\nu}+\partial_{\mu \nu}+i g \varphi_{\mu \nu}^{\prime}\right) \psi \\
& =\bar{\psi} \gamma^{\mu} \gamma^{v} D_{\mu \nu} \psi=\bar{\psi} \gamma^{\mu} \gamma^{v}\left(\partial_{\mu} \partial_{\nu}+i g \varphi_{\mu \nu}\right) \psi,
\end{aligned}
$$


by Equation (36), the gauge transformation of gravity is

$$
\phi_{\mu v}^{\prime}=\phi_{\mu v}+\frac{1}{g}\left(\partial_{\nu} \gamma(x) \partial_{\mu}+\partial_{\mu} \partial_{v} \gamma(x)+\partial_{\mu} \gamma(x) \partial_{v}-i \partial_{\mu} \gamma(x) \partial_{\nu} \gamma(x)\right),
$$

Multiplying Equation (15) by $\left(i \gamma^{v} \partial_{\mu}+m+\varepsilon / m \gamma^{\mu} \gamma^{v} \partial_{\mu} \partial_{v}\right)$ at left, we obtain

$$
\begin{aligned}
{[} & \left.\left(i \gamma^{\mu} \partial_{\mu}+m\right)+\varepsilon / m \gamma^{\rho} \gamma^{\sigma} \partial^{\rho} \partial^{\sigma}\right]\left[\left(i \gamma^{v} \partial_{v}-m\right)-\varepsilon / m \gamma^{\rho^{\prime}} \gamma^{\sigma^{\prime}} \partial_{\rho^{\prime}} \partial_{\sigma^{\prime}}\right] \psi \\
= & {\left[\left(i \gamma^{\mu} \partial_{\mu}+m\right)\left(i \gamma^{v} \partial_{v}-m\right)-\varepsilon / m\left(i \gamma^{\mu} \partial_{\mu}+m\right) \gamma^{\rho^{\prime}} \gamma^{\sigma^{\prime}} \partial_{\rho^{\prime}} \partial_{\sigma^{\prime}}\right.} \\
& \left.+\varepsilon / m\left(i \gamma^{v} \partial_{v}-m\right) \gamma^{\rho} \gamma^{\sigma} \partial_{\rho} \partial_{\sigma}-\varepsilon^{2} / m^{2} \gamma^{\rho} \gamma^{\sigma} \gamma^{\rho^{\prime}} \gamma^{\sigma^{\prime}} \partial_{\rho} \partial_{\sigma} \partial_{\rho^{\prime}} \partial_{\sigma^{\prime}}\right] \psi \\
= & {\left[\left(i \gamma^{\mu} \partial_{\mu}+m\right)\left(i \gamma^{v} \partial_{v}-m\right)-i \varepsilon / m \gamma^{\mu} \partial_{\mu} \gamma^{\rho^{\prime}} \gamma^{\sigma^{\prime}} \partial_{\rho^{\prime}} \partial_{\sigma^{\prime}}+i \varepsilon / m \gamma^{v} \partial_{v} \gamma^{\rho} \gamma^{\sigma} \partial_{\rho} \partial_{\sigma}(38\right.} \\
& \left.-\varepsilon \gamma^{\rho^{\prime}} \gamma^{\sigma^{\prime}} \partial_{\rho^{\prime}} \partial_{\sigma^{\prime}}-\varepsilon \gamma^{\rho} \gamma^{\sigma} \partial_{\rho} \partial_{\sigma}-\varepsilon^{2} / m^{2} \gamma^{\rho} \gamma^{\sigma} \gamma^{\rho^{\prime}} \gamma^{\sigma^{\prime}} \partial_{\rho} \partial_{\sigma} \partial_{\rho^{\prime}} \partial_{\sigma^{\prime}}\right] \psi \\
= & {\left[\square-m^{2}-2 \varepsilon \gamma^{\rho} \gamma^{\sigma} \partial_{\rho} \partial_{\sigma}-\varepsilon^{2} / m^{2} \gamma^{\rho} \gamma^{\sigma} \gamma^{\rho^{\prime}} \gamma^{\sigma^{\prime}} \partial_{\rho} \partial_{\sigma} \partial_{\rho^{\prime}} \partial_{\sigma^{\prime}}\right] \psi } \\
= & {\left[\square-m^{2}\right] \psi }
\end{aligned}
$$

it becomes $K-G$ equation. So, the Equation (15) is a relativistic field equation, and we can prove it is Lorentz covariant.

The Lorentz transformation is

$$
x_{\mu}^{\prime}=\Lambda_{\mu v} x^{v},
$$

and its infinitesimal transformation is

$$
\Lambda_{\mu v}=g_{\mu v}+\omega_{\mu v},
$$

where $\omega_{\mu v}$ is a infinitesimal parameter, the complete set of transformations $\Lambda_{\mu \nu}$ are called Lorentz group. At the Lorentz transformation (39), the transformation of Dirac spinor field $\psi(x)$ is

$$
\psi^{\prime}\left(x^{\prime}\right)=L(\Lambda) \psi(x),
$$

where $L(\Lambda)=\exp \left(-\frac{i}{4} \sigma^{\mu v} \Lambda_{\mu v}\right)$ is the representation of the Lorentz group for the spinor field $\psi(x), \sigma^{\mu v}=\frac{i}{2}\left(\gamma^{\mu} \gamma^{v}-\gamma^{v} \gamma^{\mu}\right), \gamma^{\mu}, \gamma^{v}$ are the Dirac matrices, and there are the following transformation relations:

$$
\begin{aligned}
L(\omega) \gamma^{v} L^{-}(\omega) & =\Lambda^{-}(\omega)_{\sigma}^{v} \gamma^{\sigma}, \\
\partial_{v} & =\partial_{\mu}^{\prime} \Lambda_{v}^{\mu}, \\
\partial_{\mu}^{\prime} & =\partial_{v} \Lambda_{\mu}^{-v},
\end{aligned}
$$

making the Lorentz transformation to Equation (15), it is

$$
\begin{aligned}
& L\left(i \gamma^{v} \partial_{v}-m-\frac{\varepsilon}{m} \gamma^{\mu} \gamma^{v} \partial_{\mu} \partial_{v}\right) \psi(x) \\
& =\left[i\left(L \gamma^{v} L^{-}\right) \partial_{v}-m-\frac{\varepsilon}{m}\left(L \gamma^{\mu} L^{-}\right)\left(L \gamma^{v} L^{-}\right) \partial_{\mu} \partial_{v}\right] L \psi(x) \\
& =\left[i \Lambda_{\mu}^{-v} \gamma^{\mu} \partial_{v}-m-\frac{\varepsilon}{m} \Lambda_{\rho}^{-\mu} \gamma^{\rho} \Lambda_{\sigma}^{-v} \gamma^{\sigma} \partial_{\mu} \partial_{v}\right] \psi^{\prime}\left(x^{\prime}\right) \\
& =\left(i \gamma^{\mu} \partial_{\mu}^{\prime}-m-\frac{\varepsilon}{m} \gamma^{\rho} \gamma^{\sigma} \partial_{\rho}^{\prime} \partial_{v}^{\prime}\right) \psi^{\prime}\left(x^{\prime}\right) \\
& =0,
\end{aligned}
$$


It has the same equation form at the Lorentz transformation, i.e., Equation (15) is the Lorentz covariant. For the $K$ - $G$ particle of spin $s=0$, its field equation is

$$
\left(\square+m^{2}\right) \phi=0
$$

and its Lagrangean density is

$$
\mathrm{L}=\frac{1}{2}\left(\partial_{\mu} \varphi \partial^{\mu} \varphi-m^{2} \varphi^{2}\right),
$$

In order to describe the gravity interaction, the Equation (46) should be modified as

$$
\left(\square+m^{2}+\lambda \beta^{\mu} \beta^{v} \partial_{\mu} \partial_{v}\right) \phi=0,
$$

where $\lambda$ is the dimensionless constant, $\beta^{\mu}$ and $\beta^{v}$ are four-dimensional constant vectors. Since the gravity is very weak, the contribution of the new added term $\lambda \beta^{\mu} \beta^{v} \partial_{\mu} \partial_{v}$ should be very small, there is

$$
\begin{gathered}
\lambda \ll m^{2}, \\
\mathrm{~L}=\frac{1}{2}\left(\partial_{\mu} \varphi \partial^{\mu} \varphi-m^{2} \varphi^{2}-2 \lambda \varphi \beta^{\mu} \beta^{v} \partial_{\mu} \partial_{\nu} \varphi\right),
\end{gathered}
$$

the Lagrangean density (47) should be modified as

$$
\frac{\partial \mathrm{L}}{\partial \varphi}=-m^{2} \varphi-\lambda \beta^{\mu} \beta^{v} \partial_{\mu} \partial_{\nu} \varphi
$$

then

$$
\partial_{\mu} \frac{\partial \mathrm{L}}{\partial\left(\partial_{\mu} \phi\right)}=\partial_{\mu} \partial^{\mu} \phi=\square \phi,
$$

substituting Equations (51) and (52) into Lagrangean equation

$$
\frac{\partial \mathrm{L}}{\partial \varphi}-\partial_{\mu} \frac{\partial \mathrm{L}}{\partial \partial_{\mu} \varphi}=0 \text {. }
$$

We can obtain the Equation (48). At U(1) gauge transformations (10) and (11), the Equation (50) should be introduced the electromagnetism and gravity gauge fields (18) and (19), and the gravity gauge transformation (37).

\section{The Equation of Gravity Field in Minkowski Spacetime}

In Section 2, we introduce gravity field and gravity gauge transformation. In the following, we should give the equation of gravity field, which is spin $s=2$ massless field. The spin $s=2$ massless field $\phi^{\mu \nu}$ generated by the energy momentum tensor $T^{\mu \nu}$ from a object, particle or a group of particles, they are symmetrical tensors

$$
\begin{gathered}
\phi^{\mu v}=\phi^{v \mu}, \\
T^{\mu v}=T^{v \mu},
\end{gathered}
$$

We construct the following independent tensors for the spin $s=2$ massless 
gravity field $\phi^{\mu \nu}$, they are

$$
\square \varphi^{\mu \nu}, \partial_{\lambda} \partial^{\mu} \phi^{\nu \lambda}, \partial_{\lambda} \partial^{v} \varphi^{\mu \lambda}, \partial^{\mu} \partial^{v} g_{\rho \sigma} \phi^{\rho \sigma}, g^{\mu v} \square g_{\rho \sigma} \varphi^{\rho \sigma}, g^{\mu \nu} \partial_{\rho} \partial_{\sigma} \phi^{\rho \sigma},
$$

the general tensor of gravity should be their linear combination, and satisfy equation

$$
\square \varphi^{\mu \nu}+a_{1}\left(\partial_{\lambda} \partial^{\mu} \varphi^{\nu \lambda}+\partial_{\lambda} \partial^{\nu} \varphi^{\mu \lambda}\right)+a_{2} \partial^{\mu} \partial^{\nu} \varphi+a_{3} g^{\mu \nu} \square \varphi+a_{4} g^{\mu \nu} \partial_{\rho} \partial_{\sigma} \varphi^{\rho \sigma}=k T^{\mu \nu},
$$

where $k$ is the gravitational constant, and $\phi=g_{\rho \sigma} \phi^{\rho \sigma}$. By derivative $\partial_{\mu}$, we have

$$
\begin{aligned}
& \square \partial_{\mu} \varphi^{\mu v}+a_{1}\left(\partial_{\lambda} \square \varphi^{\nu \lambda}\right)+a_{1} \partial_{\lambda} \partial_{\mu} \partial^{v} \varphi^{\mu v}+a_{2} \partial^{v} \square \varphi \\
& +a_{3} g^{\mu v} \square \partial_{\mu} \varphi+a_{4} g^{\mu v} \partial_{\mu} \partial_{\rho} \partial_{\sigma} \varphi^{\rho \sigma}=k \partial_{\mu} T^{\mu v},
\end{aligned}
$$

i.e.,

$$
\square \partial_{\mu} \varphi^{\mu v}\left(1+a_{1}\right)+\left(a_{1}+a_{4}\right) \partial^{v} \partial_{\rho} \partial_{\sigma} \varphi^{\rho \sigma}+\left(a_{2}+a_{3}\right) \square \partial^{v} \varphi=k \partial_{\mu} T^{\mu v},
$$

the source tensor $T^{\mu \nu}$ is conservative, it is

$$
\partial_{\mu} T^{\mu v}=0,
$$

by Equation (59) and (60), we get

$$
a_{1}=-1, a_{4}=1, a_{2}=-a_{3},
$$

and Equation (57) becomes

$$
\square \varphi^{\mu \nu}-\left(\partial_{\lambda} \partial^{\mu} \varphi^{\nu \lambda}+\partial_{\lambda} \partial^{v} \varphi^{\mu \lambda}\right)+a_{2} \partial^{\mu} \partial^{v} \varphi-a_{2} g^{\mu v} \square \varphi+g^{\mu v} \partial_{\rho} \partial_{\sigma} \varphi^{\rho \sigma}=k T^{\mu v},
$$

take $a_{2}=1$, we obtain

$$
\square \varphi^{\mu \nu}-\left(\partial_{\lambda} \partial^{\mu} \varphi^{\nu \lambda}+\partial_{\lambda} \partial^{v} \varphi^{\mu \lambda}\right)+\partial^{\mu} \partial^{v} \varphi-g^{\mu v} \square \varphi+g^{\mu v} \partial_{\rho} \partial_{\sigma} \varphi^{\rho \sigma}=k T^{\mu \nu},
$$

if we choose the following gauge condition

$$
\partial_{\mu}\left(\phi^{\mu v}-\frac{1}{2} g^{\mu v} \phi\right)=0,
$$

i.e.,

$$
\partial_{\mu} \phi^{\mu v}=\frac{1}{2} g^{\mu v} \partial_{\mu} \phi=\frac{1}{2} \partial^{v} \phi,
$$

then

$$
\begin{gathered}
\partial_{\lambda} \partial^{\mu} \phi^{\nu \lambda}=\partial^{\mu} \partial_{\lambda} \phi^{\nu \lambda}=\frac{1}{2} \partial^{\mu} \partial^{v} \phi, \\
\partial_{\lambda} \partial^{v} \phi^{\mu \lambda}=\partial^{v} \partial_{\lambda} \phi^{\mu \lambda}=\frac{1}{2} \partial^{v} \partial^{\mu} \phi, \\
\partial^{\mu} \partial^{v} \varphi=2 \partial_{\mu} \partial^{\mu} \varphi^{\mu \nu}=2 \square \varphi^{\mu \nu}=\partial^{\mu} \partial^{v} \varphi, \\
g^{\mu v} \partial_{\rho} \partial_{\sigma} \varphi^{\rho \sigma}=g^{\mu v} \partial_{\rho}\left(\partial_{\sigma} \varphi^{\rho \sigma}\right)=g^{\mu \nu} \partial_{\rho} \frac{1}{2} \partial^{\rho} \varphi=\frac{1}{2} g^{\mu v} \square \varphi,
\end{gathered}
$$

substituting Equations (66)-(69) into Equations (63), it becomes

$$
\square\left(\varphi^{\mu \nu}-\frac{1}{2} g^{\mu \nu} \varphi\right)=k T^{\mu \nu},
$$


defining the new tensor $\Phi^{\mu v}$ as the gravity field tensor, it is

$$
\Phi^{\mu v}=\phi^{\mu v}-\frac{1}{2} g^{\mu v} \phi,
$$

the Equation (64) and (70) become

$$
\square \Phi^{\mu v}=k T^{\mu v},
$$

and

$$
\partial_{\mu} \Phi^{\mu v}=0,
$$

the Equation (72) is the gravity field equation including source, and the gauge condition is (73). In the absence of sources, the free gravity field and the gauge condition become

$$
\square \Phi^{\mu v}=0,
$$

and

$$
\partial_{\mu} \Phi^{\mu v}=0,
$$

the Lagrangean density of Equation (72) is

$$
L=\partial_{\mu} \Phi^{\mu v} \cdot \partial^{\mu} \Phi_{\mu v},
$$

then

$$
\begin{gathered}
\frac{\partial L}{\partial \partial_{\mu} \Phi^{\mu \nu}}=\partial^{\mu} \Phi_{\mu v}, \\
\frac{\partial L}{\partial_{\mu} \Phi_{\mu v}}=0
\end{gathered}
$$

substituting Equations (77) and (78) into the Lagrangean equation

$$
\frac{\partial L}{\partial_{\mu} \Phi_{\mu v}}-\partial_{\mu} \frac{\partial L}{\partial \partial_{\mu} \Phi^{\mu \nu}}=0
$$

we can obtain the free gravity field equation

$$
\square \Phi^{\mu v}=0,
$$

the Equation (80) has the plane wave solution, which is the gravitational wave, it is

$$
\Phi^{\mu v}(\boldsymbol{r}, t)=\Phi_{0} e^{i(\boldsymbol{k} \cdot \boldsymbol{r}-\omega t)} e^{\mu v},
$$

where $\Phi_{0}$ is the amplitude, $\boldsymbol{k}(\omega)$ is the wave vector (frequency), and $e_{\mu \nu}$ is the second order polarization tensor. The general solution of Equation (80) is

$$
\Phi^{\mu \nu}(\boldsymbol{r}, t)=\sum_{i} \sum_{\mu, v} \Phi_{i 0} e^{i(\boldsymbol{k} \cdot \boldsymbol{r}-\omega t)} e^{\mu \nu}
$$

In the Newton gravity, the Newtonian potential $\phi$ satisfies Poisson equation

$$
\nabla^{2} \varphi=4 \pi G \rho_{0},
$$

where $G$ is the gravity constant, and $\rho_{0}$ is mass density, there is,

$$
\rho_{0}=\frac{T^{00}}{c^{2}},
$$


the Equation (83) can be written as

$$
\nabla^{2} \varphi=\frac{4 \pi G}{c^{2}} T^{00},
$$

When $\mu=\rho=0$, and a object or particle is at rest, the Equation (72) becomes

$$
\nabla^{2} \Phi^{00}=k T^{00},
$$

comparing Equation (85) with (86), we have

$$
\begin{gathered}
\Phi^{00}=\varphi, \\
k=\frac{4 \pi G}{c^{2}},
\end{gathered}
$$

then the gravity field Equation (72) becomes

$$
\square \Phi^{\mu v}=\frac{4 \pi G}{c^{2}} T^{\mu v},
$$

with the gauge condition (75), there is

$$
\partial_{\mu} \square \Phi^{\mu v}=\square \partial_{\mu} \Phi^{\mu v}=0,
$$

For the closed system, the energy momentum tensor of the whole system satisfies the conservation law, it is

$$
\frac{\partial}{\partial x^{\mu}} T^{\mu v}=\partial_{\mu} T^{\mu v}=0
$$

The gravity field Equation (89) satisfies the condition of energy momentum conservation.

For a object or particle, its mass $m$, mass density $\rho$, velocity $u^{\mu}$, energy momentum tensor $T^{\mu v}=\rho u^{\mu} u^{v}$, and its gravity field equation is

$$
\square \Phi^{\mu v}=\frac{4 \pi G}{c^{2}} \rho u^{\mu} u^{v},
$$

when the object or particle is at rest, the velocity component $u^{i}=0(i=1,2,3)$, the Equation (89) becomes

$$
\nabla^{2} \Phi^{00}=\frac{4 \pi G}{c^{2}} T^{00}=4 \pi G \rho_{0},
$$

and

$$
\nabla^{2} \Phi^{0 i}=\nabla^{2} \Phi^{i 0}=\nabla^{2} \Phi^{i j}=0,
$$

where $\rho_{0}$ is the rest mass density of object or particle, the solutions of Equations (93) and (94) are

$$
\begin{gathered}
\Phi^{00}=-G \frac{m_{0}}{r}, \\
\Phi^{i 0}=\Phi^{0 i}=c_{1}, \\
\Phi^{i j}=c_{2},
\end{gathered}
$$

where $c_{1}$ and $c_{2}$ are the constants, and $\Phi^{00}$ is the Newtonian gravitational potential. In the nonrelativistic limit, the gravity field Equation (89) becomes the 
Newtonian gravity equation.

For the spin Dirac field, the total Lagrangean density is

$$
L=\bar{\psi}\left(i \gamma^{\mu} D_{\mu}-m-\frac{\varepsilon}{m} \gamma^{\mu} \gamma^{v} D_{\mu v}\right) \psi+\partial_{\mu} \Phi^{\mu v} \cdot \partial^{\mu} \Phi_{\mu v},
$$

For the spin $s=0, K-G$ field, the total Lagrangean density is,

$$
L=\frac{1}{2}\left(D_{\mu} \varphi D^{\mu} \varphi-m^{2} \varphi^{2}-2 \lambda \varphi \beta^{\mu} \beta^{v} D_{\mu \nu} \varphi\right)+\partial_{\mu} \Phi^{\mu v} \cdot \partial^{\mu} \Phi_{\mu v},
$$

The Equations (98) and (99) can be described electroweak interaction, strong interaction and gravity interaction for spin $s=1 / 2$ and $s=0$ particle and field.

The free gravity field Equation (80) is a tensor equation, which is Lorentz covariant. In the following, we shall prove the Lagrangean density (76) is $U(1)$ gauge invariant.

At the transformation (37), the gravity field gauge transformation is,

$$
\begin{aligned}
& \Phi_{\mu v}^{\prime}=\varphi_{\mu v}^{\prime}-\frac{1}{2} g_{\mu v} \varphi^{\prime} \\
& =\varphi_{\mu \nu}+\frac{1}{g}\left(\partial_{\nu} \gamma(x) \partial_{\mu}+\partial_{\mu} \partial_{\nu} \gamma(x)+\partial_{\mu} \gamma(x) \partial_{\nu}-i \partial_{\mu} \gamma(x) \partial_{\nu} \gamma(x)\right) \\
& -\frac{1}{2} g_{\mu \nu} g_{\rho \sigma}\left(\varphi^{\rho \sigma}+\frac{1}{g}\left(\partial^{\sigma} \gamma(x) \partial^{\rho}+\partial^{\rho} \partial^{\sigma} \gamma(x)+\partial^{\rho} \gamma(x) \partial^{\sigma}-i \partial^{\rho} \gamma(x) \partial^{\sigma} \gamma(x)\right)\right) \\
& =\varphi_{\mu v}+\frac{1}{g}\left(\partial_{\nu} \gamma(x) \partial_{\mu}+\partial_{\mu} \partial_{v} \gamma(x)+\partial_{\mu} \gamma(x) \partial_{v}-i \partial_{\mu} \gamma(x) \partial_{v} \gamma(x)\right) \\
& -\frac{1}{2} g_{\mu v}\left(\varphi+\frac{1}{g}\left(2 \partial_{\mu} \gamma(x) \partial^{\mu}+\square \gamma(x)-i \partial_{\mu} \gamma(x) \partial^{\mu} \gamma(x)\right)\right) \\
& =\Phi_{\mu \nu}+\frac{1}{g}\left(\partial_{\nu} \gamma(x) \partial_{\mu}+\partial_{\mu} \partial_{\nu} \gamma(x)+\partial_{\mu} \gamma(x) \partial_{\nu}-i \partial_{\mu} \gamma(x) \partial_{\nu} \gamma(x)\right) \\
& -\frac{1}{2} g_{\mu \nu} \frac{1}{g}\left(2 \partial_{\mu} \gamma(x) \partial^{\mu}+\square \gamma(x)-i \partial_{\mu} \gamma(x) \partial^{\mu} \gamma(x)\right),
\end{aligned}
$$

then

$$
\begin{aligned}
\partial_{\mu} \Phi_{\mu v}^{\prime}= & \partial^{\mu} \Phi_{\mu v}-\frac{1}{g} \partial^{\mu}\left(\partial_{\nu} \gamma(x) \partial_{\mu}+\partial_{\mu} \partial_{v} \gamma(x)+\partial_{\mu} \gamma(x) \partial_{v}-i \partial_{\mu} \gamma(x) \partial_{v} \gamma(x)\right) \\
& -\frac{1}{g} \partial_{v}\left(\partial_{\mu} \gamma(x) \partial^{\mu}+\frac{1}{2} \square \gamma(x)-\frac{1}{2} i \partial_{\mu} \gamma(x) \partial^{\mu} \gamma(x)\right) \\
= & \partial^{\mu} \Phi_{\mu v}+\partial^{\mu} F_{\mu v}+\partial_{\nu} G,
\end{aligned}
$$

where $\phi^{\prime}=g_{\rho \sigma} \phi^{\prime \rho \sigma}$, and

$$
\begin{gathered}
F_{\mu v}=-\frac{1}{g}\left(\partial_{v} \gamma(x) \partial_{\mu}+\partial_{\mu} \partial_{v} \gamma(x)+\partial_{\mu} \gamma(x) \partial_{\nu}-i \partial_{\mu} \gamma(x) \partial_{\nu} \gamma(x)\right), \\
G=-\frac{1}{g}\left(\partial_{\mu} \gamma(x) \partial^{\mu}+\frac{1}{2} \square \gamma(x)-\frac{1}{2} i \partial_{\mu} \gamma(x) \partial^{\mu} \gamma(x)\right)
\end{gathered}
$$

similarly, we have 


$$
\partial_{\mu} \Phi^{\prime \mu v}=\partial_{\mu} \Phi^{\mu v}+\partial_{\mu} F^{\mu v}+\partial^{v} G
$$

the gauge transformation of Lagrangean density is

$$
\begin{aligned}
& L^{\prime}=\partial_{\mu} \Phi^{\prime \mu v} \cdot \partial^{\mu} \Phi_{\mu v}^{\prime} \\
&=\left(\partial_{\mu} \Phi^{\mu v}+\partial_{\mu} F^{\mu v}+\partial^{v} G\right) \cdot\left(\partial^{\mu} \Phi_{\mu v}+\partial^{\mu} F_{\mu v}+\partial_{v} G\right) \\
&=\left(\partial_{\mu} \Phi^{\mu v}\right) \cdot\left(\partial^{\mu} \Phi_{\mu v}\right)+\left(\partial_{\mu} \Phi^{\mu v}\right) \cdot\left(\partial^{\mu} F_{\mu v}\right)+\left(\partial_{\mu} \Phi^{\mu v}\right) \cdot \partial_{\nu} G \\
&+\left(\partial_{\mu} F^{\mu v}\right) \cdot\left(\partial^{\mu} \Phi_{\mu v}\right)+\left(\partial_{\mu} F^{\mu v}\right) \cdot\left(\partial^{\mu} F_{\mu v}\right)+\left(\partial_{\mu} F^{\mu v}\right) \cdot \partial_{\nu} G \\
&+\left(\partial^{v} G\right) \cdot\left(\partial^{\mu} \Phi_{\mu v}\right)+\left(\partial^{v} G\right) \cdot\left(\partial^{\mu} F_{\mu v}\right)+\left(\partial^{v} G\right) \cdot \partial_{v} G,
\end{aligned}
$$

and the variation of action is

$$
\begin{aligned}
\delta S^{\prime}= & \delta \int L^{\prime} \mathrm{d}^{4} x=\delta \int\left(\partial_{\mu} \Phi^{\prime \mu v} \cdot \partial^{\mu} \Phi_{\mu v}^{\prime}\right) \mathrm{d}^{4} x \\
= & \delta \int\left(\left(\partial_{\mu} \Phi^{\mu v}\right) \cdot\left(\partial^{\mu} \Phi_{\mu v}\right)+\left(\partial_{\mu} \Phi^{\mu v}\right) \cdot\left(\partial^{\mu} F_{\mu v}\right)+\left(\partial_{\mu} \Phi^{\mu v}\right) \cdot \partial_{v} G\right. \\
& +\left(\partial_{\mu} F^{\mu v}\right) \cdot\left(\partial^{\mu} \Phi_{\mu v}\right)+\left(\partial_{\mu} F^{\mu v}\right) \cdot\left(\partial^{\mu} F_{\mu v}\right)+\left(\partial_{\mu} F^{\mu v}\right) \cdot \partial_{v} G \\
& \left.+\left(\partial^{v} G\right) \cdot\left(\partial^{\mu} \Phi_{\mu v}\right)+\left(\partial^{v} G\right) \cdot\left(\partial^{\mu} F_{\mu v}\right)+\left(\partial^{v} G\right) \cdot \partial_{v} G\right) \mathrm{d}^{4} x \\
= & \delta \int\left(\partial_{\mu} \Phi^{\mu v} \cdot \partial^{\mu} \Phi_{\mu v}\right) \mathrm{d}^{4} x=\delta \int L \mathrm{~d}^{4} x=\delta S .
\end{aligned}
$$

In Equation (106), the variation is to field $\Phi^{\mu v}$, and the Lagrangean density is equivalent when it adds a term of four-dimension divergence. Since the variation of action is equivalent before and after the $U(1)$ gauge transformation, the Lagrangean density of gravity field (76) is $U(1)$ gauge invariant. By the Lagrangean density of gravity field (76) and the gravity field Equation (89), we can quantize the gravitational field with the canonical quantization or path integral quantization, which should be studied in the future.

\section{Conclusion}

In this paper, we have proposed the gauge theory of gravity. In Dirac equation and $K$ - $G$ equation, they have introduced the vector gauge field, such as electroweak and strong interaction gauge field, which are vector gauge fields, and have not introduced the gravity gauge field. In order to introduce the gravity gauge field, we should add a term of partial derivative $\partial_{\mu} \partial_{v}$ in Dirac equation and $K-G$ equation. At the $U(1)$ gauge transformation, the gravity gauge field should be introduced naturally. Otherwise, we give the equation of gravity tensor field at the flat Minkowski spacetime, and further prove the gravity field equation is the Lorentz covariant and gauge invariant. The gravity theory can be quantized and can be unified with the electroweak and strong interaction a new gauge group $U(1) \otimes S U(2) \otimes S U(3)$, which should be studied in the future.

\section{Acknowledgements}

This work was supported by the Research innovation project of Jilin Normal University (no. 201627) and the Scientific and Technological Development 
Foundation of Jilin Province (no. 20130101031JC).

\section{References}

[1] Einstein, A. (1916) Die Grundlage der allgemeinen Relativitätstheorie. Annalen der Physik, 49, 769. https://doi.org/10.1002/andp.19163540702

[2] Einstein, A. (1913) Entwurf einer verallgemeinerten Relativitätstheorie u.einer Theorie der Gravitation. Zeitschrift für angewandte Mathematik und Physik, 62, 225.

[3] MacDowell, S.W. and Mansouri, F. (1977) Unified Geometric Theory of Gravity and Supergravity. Physical Review Letters, 38, 739.

https://doi.org/10.1103/PhysRevLett.38.739

[4] Wise, D.K. (2010) MacDowell-Mansouri Gravity and Cartan Geometry. Classical and Quantum Gravity, 27, Article ID: 155010. https://doi.org/10.1088/0264-9381/27/15/155010

[5] Hiesmayr, B.C., Di Domenico, A., Curceanu, C., Gabriel, A., Huber, M., Larsson, J.A. and Moscal, P. (2012) Revealing Bell's Nonlocality for Unstable Systems in High Energy Physics. The European Physical Journal C, 72, 1856. https://doi.org/10.1140/epjc/s10052-012-1856-X

[6] Amelino-Camelia, G., Ellis, J., Mavromatos, N.E., Nanopoulos, D.V. and Sarkar, S. (1998) Tests of Quantum Gravity from Observations of $\gamma$-Ray Bursts. Nature, 393, 763-765. https://doi.org/10.1038/31647

[7] Amelino-Camelia, G. (1999) Gravity-Wave Interferometers as Quantum-Gravity Detectors. Nature, 398, 216-218. https://doi.org/10.1038/18377

[8] Alfaro, J., Morales-Tecotl, H.A. and Urrutia, L.F. (2000) Quantum Gravity Corrections to Neutrino Propagation. Physical Review Letters, 84, 2318. https://doi.org/10.1103/PhysRevLett.84.2318

[9] Jacobson, T., Liberati, S. and Mattingly, D. (2003) A Strong Astrophysical Constraint on the Violation of Special Relativity by Quantum Gravity. Nature, 424, 1019-1021. https://doi.org/10.1038/nature01882

[10] Myers, R.C. and Pospelov, M. (2003) Ultraviolet Modifications of Dispersion Relations in Effective Field Theory. Physical Review Letters, 90, Article ID: 211601. https://doi.org/10.1103/PhysRevLett.90.211601

[11] Amelino-Camelia, G. and Lammerzahl, C. (2004) Quantum-Gravity-Motivated Lorentz-Symmetry Tests with Laser Interferometers. Classical and Quantum Gravity, 21, 899. https://doi.org/10.1088/0264-9381/21/4/010

[12] Jacob, U. and Piran, T. (2007) Neutrinos from Gamma-Ray Bursts as a Tool to Explore Quantum-Gravity-Induced Lorentz Violation. Nature Physics, 3, 87.

[13] Contaldi, C.R.., Dowker, F. and Philpott, L. (2010) Polarization Diffusion from Spacetime Uncertainty. Classical and Quantum Gravity, 27, Article ID: 172001. https://doi.org/10.1088/0264-9381/27/17/172001

[14] Botermann, B., et al. (2014) Test of Time Dilation Using Stored $\mathrm{Li}^{+}$Ions as Clocks at Relativistic Speed. Physical Review Letters, 113, Article ID: 120405. https://doi.org/10.1103/PhysRevLett.113.120405

[15] Dowdall, R.J. and Fairbairn, W.J. (2011) Observables in 3d Spinfoam Quantum Gravity with Fermions. General Relativity and Gravitation, 43, 1263-1307. https://doi.org/10.1007/s10714-010-1107-0

[16] Poplawski, N.J. (2010) Cosmology with Torsion: An Alternative to Cosmic Infla- 
tion. Physics Letters B, 694, 181.

[17] Resconi, G., Licata, I. and Corda, C. (2017) Gravity with Torsion in Non-Conservative Maxwell-Like Gauge Approach. International Journal of Geometric Methods in Modern Physics, 7, Article ID: 1750110. 